MNHMT2009-18479

\title{
FEEDSTOCK DIFFUSION AND DECOMPOSITION IN ALIGNED CARBON NANOTUBE ARRAYS
}

\author{
Rong Xiang, Erik Einarsson, Junichiro Shiomi, Shigeo Maruyama
}

The University of Tokyo, 7-3-1 Hongo, Bunkyo-ku, Tokyo 113-8656, Japan

\begin{abstract}
Feedstock diffusion and decomposition in the root growth of aligned carbon nanotube (CNT) arrays is discussed. A nondimensional modulus is proposed to differentiate catalystpoisoning controlled growth deceleration from one which is diffusion controlled. It is found that, at current stage, aligned multi-walled carbon nanotube (MWNT) arrays are usually free of feedstock diffusion resistance. However, for single-walled carbon nanotube (SWNT) arrays, since the inter-tube distance is much smaller than the mean free path of carbon source (ethanol here), high diffusion resistance is significantly limiting the growth rate. The method presented here is also able to predict the critical lengths in different chemical vapor deposition (CVD) processes from which CNT arrays begin to meet this diffusion limit, as well as the possible solutions to this diffusion caused growth deceleration. The diffusion of carbon source inside of an array becomes more important when we found ethanol undergoes severe thermal decomposition at the reaction temperature. This means, in a typical alochol CVD, hydrocarbons and radicals decomposed from ethanol may collide and react with the outer walls of SWNTs before reaching catalyst particles. We found when flow rate is low and ethanol is thoroughly decomposed, the produced SWNTs contain more soot structures than the SWNTs obtained at higher ethanol flow rate. Understanding the mass transport and reaction inside a SWNT array is helpful to synthesize longer and cleaner SWNTs.
\end{abstract}

\section{INTRODUCTION}

Vertically aligned (VA-) carbon nanotube (CNT) arrays grown on flat substrates ${ }^{[1-7]}$, in which all the nanotubes are of similar orientation and length, offer an ideal platform to study
CNT growth mechanisms and kinetics. Since $1996^{[1]}$, various chemical vapor deposition (CVD) methods, including floating catalytic $\mathrm{CVD}^{[2]}$, plasma enhanced $\mathrm{CVD}^{[3]}$ and thermal $\mathrm{CVD}^{[4]}$ have been proposed to synthesize aligned multi-walled carbon nanotube (MWNT) arrays. Lately, alcohol catalytic $\mathrm{CVD}^{[5]}$ (ACCVD), water assisted $\mathrm{CVD}^{[6]}$, microwave plasma $\mathrm{CVD}^{[7]}$, etc. are used to produce vertically aligned single-walled carbon nanotube (SWNT) arrays. These processes usually involve different catalysts, carbon sources and operation parameters, resulting in products with different morphologies and qualities. However, none of these CNT growth processes can overcome the gradual deceleration and eventual termination of growth. The ability to understand and thereby to overcome the underlying deactivation mechanisms becomes one of the most critical steps to develop nano-scale tubes into real macroscopic materials.

Recently, many groups have affirmed the root growth mode of their vertically aligned CNTs, indicating that the feedstock molecules have to diffuse through the thick CNT array, reach the substrate where catalysts are located, and then contribute to the CNT growth. ${ }^{[8-12]}$ In this bottom-up growth process, the diffusion resistance of the feedstock from the top to the root arises as an obstruction, and can act as a unique decelerating growth mechanism. At the same time, since feedstock molecules go through CNT "channels", bulk reaction during this diffusion process may also play a role on the real chemical reaction on the catalyst surface.

Here, first we confirmed the root growth mechanism in our ACCVD, which is the premise of a diffusion argument. We propose a method of using a non-dimensional modulus to quantitatively evaluate the degree of feedstock diffusion resistance (no diffusion resistance regime, transient regime, and strong diffusion limit regime). Currently available singlewalled carbon nanotubes (SWNTs) were found already in a 
strong diffusion limit region while multi-walled carbon nanotubes (MWNTs) are still free of diffusion resistance. This difference is caused by the different tube-tube spacing for various systems (channel size of a CNT film). The method presented here is also able to predict the critical lengths in different CVD processes from which carbon nanotube arrays begin to meet strong diffusion resistance, as well as the possible solutions to this diffusion caused growth deceleration. The ethanol decomposition is also calculated, because this is critical for the real gas composition around the catalyst surface. It is also important for the quality of SWNTs produced, because decomposed gas molecules collide many times with SWNT walls before reaching the catalyst surface.

\section{RESULTS AND DISSCUSION}

A classic argument in the growth of CNT is that whether the catalyst sits at the CNT-substrate interface (root growth) or moves along with a CNT (tip growth). To clarify this point, we started the CVD with ${ }^{12} \mathrm{C}$ ethanol and switch to ${ }^{13} \mathrm{C}$ one during the growth. The resulting material are VA-SWNTs with ${ }^{12} \mathrm{C}-{ }^{13} \mathrm{C}$ junctions on a flat quartz substrate, as shown in Fig. 1a.

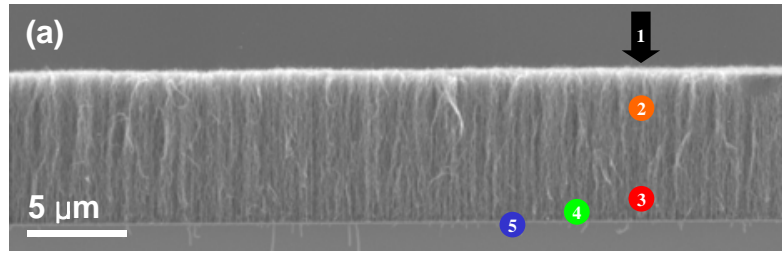

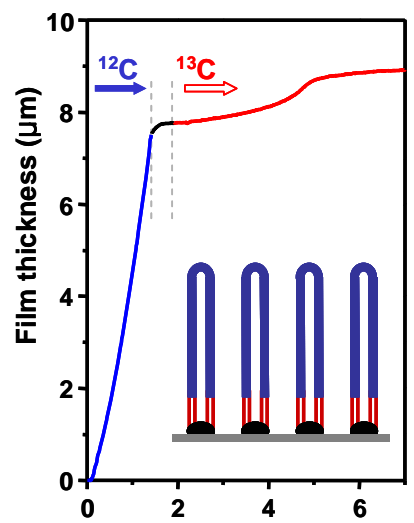

(b) CVD time ( $\mathrm{min})$

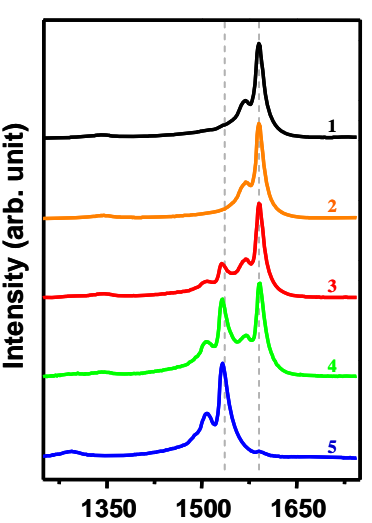

(c) Raman shift $\left(\mathrm{cm}^{-1}\right)$
Fig. 1. (a) SEM image, (b) growth curve from in situ optical absorption and (c) cross-sectional Raman spectra across $a{ }^{12} \mathrm{C}-{ }^{13} \mathrm{C}$ junction. The arrow and spots in (a) represent the positions of the incident light. The inset schematic in (b) illustrates the junction structure in the case of root growth.

Fig. 1b shows the growth curve obtained from in situ optical absorption. The entire array contains $8 \mu \mathrm{m}$ of ${ }^{12} \mathrm{C}$ and 1 $\mu \mathrm{m}$ of ${ }^{13} \mathrm{C}$ nanotubes. As the ${ }^{12} \mathrm{C}$ is fed before the ${ }^{13} \mathrm{C}$, the location of this $1 \mu \mathrm{m}{ }^{13} \mathrm{C}$ can be used to clarify whether the SWNTs are grown from the root of the array or from the tip. Raman spectra taken from the side of the array is shown in Fig 1c. It is very clear that ${ }^{13} \mathrm{C}$ only locates near the root of the
SWNT array; this is solid evidence for the root growth mechanism, shown by the schematic in the inset of Figlb. This result is also consistent with TEM observation, where most of the catalyst particles are usually found at only one edge of the array. ${ }^{[13]}$

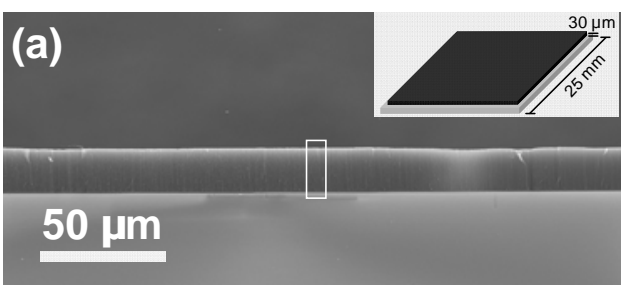

(b)

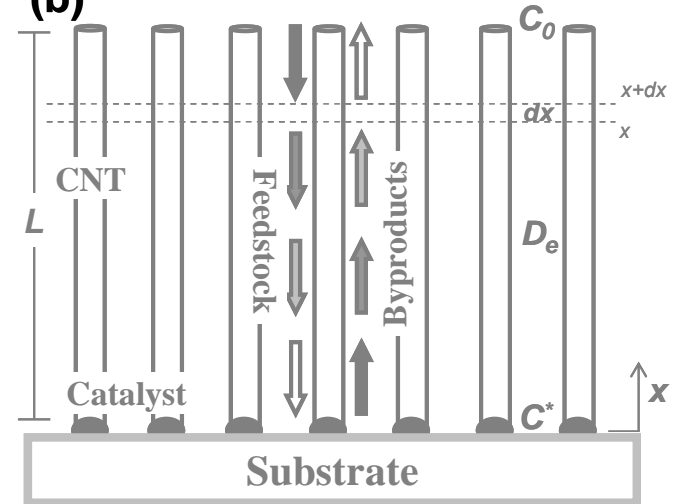

Fig. 2. (a) SEM micrograph of vertically aligned SWNT arrays from ACCVD, inset at top-right is a schematic of a CNT film on substrate, suggesting the different dimensions of film size and thickness; (b) schematic presentation describing the diffusion of feedstock as well as gas product during the root growth of CNT arrays.

After confirming the root growth mechanism, feedstock and byproduct diffusion inside VA-CNT arrays is modeled, because strong diffusion resistance of precursor molecules could be one reason for the growth deceleration of VA-CNT observed by many groups. The one dimensional diffusion model of precursor inside of film reveals the thickness of a VACNT film can be expressed as

$$
L=\sqrt{\left(\frac{D_{e}}{k_{s}}\right)^{2}+\frac{2 D_{e} C_{0}}{a} t}-\frac{D_{e}}{k_{s}}
$$

where $L$ is thickness of the CNT film, $k_{s}$ the reaction constant from starting precursor to CNTs, $D_{e}$ diffusion coefficient of precursor molecules inside of a CNT array, $a$ CNT structure related parameter, $C_{0}$ bulk gas concentration and $t$ growth time. Then the film thickness $L$ can be either This equation can be proportional to either $t$ (no diffusion limit) or $t^{1 / 2}$ (strong diffusion limit), depending on the values of $2 D_{e} C_{0} t / a$ and $D_{e} / k_{s}$. It is similar to what is widely used in silicon oxidation, the socalled "Deal-Grove" relationship ${ }^{[14]}$, as discussed previously. ${ }^{[15]}$ One can, in principle, also predict the growth curve of a CNT array provided that all the parameters listed above are known. However, a big difference between growth of a CNT array and silicon oxide is that, in most cases, the catalyst for CNT growth 
undergoes catalyst poisoning. Therefore, in CNT growth $k_{s}$ is also a time-dependent parameter, unlike in silicon oxidation, where $k_{s}$ is constant. This means the above equation only predict the ideal growth curve where catalyst activity does not decay.

We propose a non-dimensional modulus $\varphi=k_{s} L / D_{e}$ to differentiate a catalyst poisoning controlled process from a diffusion controlled one. The strength of this modulus is that simply its value tells quantitatively the diffusion resistance. The ratio between effective concentration around the catalyst and bulk concentration can be determined by this modulus only as $\eta=1 /(\varphi+1)$. At current stage, aligned MWNT arrays are usually free of feedstock diffusion limit while SWNT arrays are already suffering from a strong diffusion resistance. The details of the calculation can be found in our report, ${ }^{[16]}$ and the critical parameters determining the difference between SWNTs and MWNTs are listed in Table 1. The method presented here is also able to predict in various CVD processes the critical lengths from which CNT arrays begin to meet strong diffusion resistance, as well as the possible solutions to this diffusion caused growth deceleration. This conclusion is consistent with currently available case studies. We also obtained $100 \mu \mathrm{m}$ long VA-SWNTs from our ACCVD process, which further supports our calculation. ${ }^{[17]}$

Table 1. System parameters and as-calculated $\varphi$ and $\eta$.

\begin{tabular}{cccccccc}
\hline \hline Parameters & Abb & Unit & A- & S- & P- & T- & F- \\
\hline Temperature & $T$ & $(\mathrm{~K})$ & 1073 & 1023 & 873 & 1023 & 1073 \\
Molecular & $M$ & $(-)$ & 46 & 28 & 16 & 28 & 84 \\
Density & - & $(\mathrm{g} / \mathrm{c}$ & 0.041 & 0.037 & 0.067 & 0.014 & 0.082 \\
CNT Diameter & - & $(\mathrm{nm})$ & 2 & 3 & 2 & 10 & 29 \\
Number density & - & $(\mathrm{m}-$ & $8.5 \mathrm{E} 15$ & $5.2 \mathrm{E} 15$ & $1.4 \mathrm{E} 16$ & $3 \mathrm{E} 14$ & $2.1 \mathrm{E} 13$ \\
Porosity & $\rho$ & $(-)$ & 0.973 & 0.963 & 0.956 & 0.976 & 0.986 \\
Inter-tube & - & $(\mathrm{nm})$ & 8.8 & 10.9 & 6.5 & 48 & 189 \\
Mean free path & $\lambda$ & $(\mathrm{nm})$ & 16000 & 206 & 5500 & 196 & 91 \\
Growth rate & - & $(\mathrm{m} / \mathrm{s}$ & $2 \mathrm{E}-7$ & $3.75 \mathrm{E}-6$ & $5 \mathrm{E}-8$ & $1.2 \mathrm{E}-6$ & $5 \mathrm{E}-7$ \\
Reaction & $k_{s}$ & $(\mathrm{~m} / \mathrm{s}$ & $2.4 \mathrm{E}-3$ & $9.2 \mathrm{E}-3$ & $5.7 \mathrm{E}-3$ & $1.2 \mathrm{E}-4$ & $3.5 \mathrm{E}-4$ \\
Diffusion & $D_{e}$ & $(\mathrm{~cm})^{2}$ & 0.013 & 0.020 & 0.015 & 0.085 & 0.169 \\
Length & $L$ & $(\mathrm{~mm}$ & 0.03 & 2.5 & 2.5 & 0.4 & 2 \\
Proposed & $\varphi$ & $(-)$ & 0.054 & 11.3 & 9.7 & 0.0057 & 0.042 \\
Effective factor & $\eta$ & $(-)$ & 0.949 & 0.081 & 0.093 & 0.994 & 0.960 \\
\hline \hline
\end{tabular}

As most feedstock species undergo some degree of thermal decomposition before reaching the catalyst, understanding the reaction pathway and the actual reacting species are important in improving understanding of the CVD processes and control over the final SWNT product. The primary byproducts of ethanol thermal decomposition at $800{ }^{\circ} \mathrm{C}$ are ethylene and water, but existing of small amounts of acetylene in also predicted by CHEMKIN (Fig. 3c) and further supported experimentally by our FT-IR results. When we change the flow rate of ethanol but keep the temperature and pressure the same, Fig. 3a shows the gas temperature doesn't change much (only pre-decomposition time changes) but growth curve behavior distinctively (Fig. 3b). Since acetylene has shown to be highly active, even in very low concentration, the apparent activity of ethanol may come partly from acetylene. Though we have confirmed not all SWNTs are grown from the decomposed gases like acetylene or ethylene, the gas composition is found critical for the growth behavior and also quality of the SWNTs obtained. Usually in the lowflow and no-flow cases, when ethanol is thoroughly decomposed, the SWNTs contain more amorphous carbon than those formed more directly from ethanol. This is evidenced by direct TEM observation. A possible explanation is as follows. As shown in Tab. 1, the tube-tube distance is comparable to the mean free path of ethanol in our ACCVD. This means gas molecules need to collide with SWNT walls many times before reaching a catalyst located at SWNT-substrate surface. Among these collisions, decomposed species like acetylene or some radicals may have much higher chance (comparing to ethanol) to react with the wall. Therefore, there is more carbon deposition. This emphasizes the importance of keeping alcohol as the main precursor for producing high purity SWNTs. ${ }^{[18]}$ Further work is needed to fully understand this phenomenon. Some related work can be found in our other reports. ${ }^{[19-21]}$

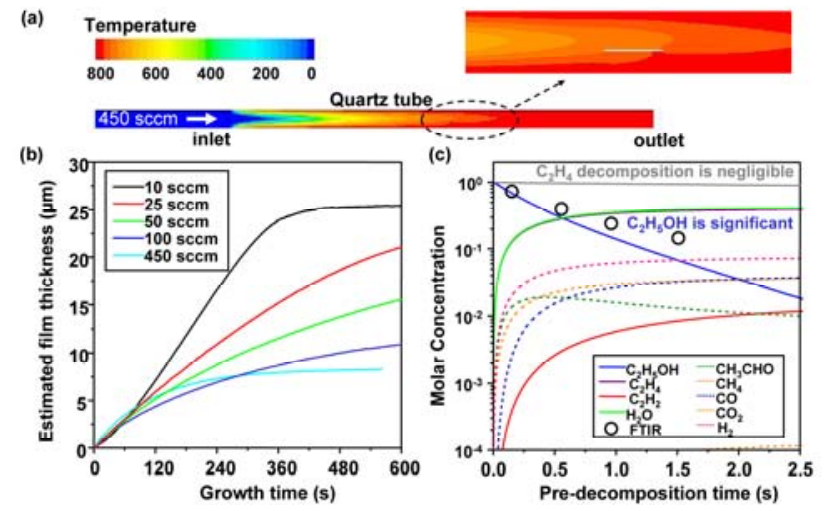

Fig. 3. (a) Temperature distribution inside the quartz tube during CVD (b) Growth curves at $800{ }^{\circ} \mathrm{C}$ for different ethanol flow rates show a change for slow flow rates. (c) Ethanol decomposition curves calculated by CHEMKIN, and experimentally measured ethanol concentrations (circles) by FTIR spectroscopy.

\section{CONCLUSIONS}

We have confirmed the root growth mechanism in our ACCVD using isotope labeling. A non-dimensional modulus is proposed to quantitatively evaluate the degree of feedstock diffusion resistance. Currently available SWNTs were found already in a strong diffusion limit region while MWNTs are still free of diffusion resistance. The method presented here is also able to predict the critical lengths in different CVD processes from which carbon nanotube arrays begin to meet strong diffusion resistance, as well as the possible solutions to this diffusion caused growth deceleration. The ethanol decomposition is also calculated, because this is critical for the real gas composition around the catalyst surface. It is also important for the quality of SWNTs produced, probably because decomposed gas molecules collide many times with SWNT walls before reaching the catalyst surface. 


\section{ACKNOWLEDGMENTS}

Part of this work was financially supported by Grants-inAid for Scientific Research (19206024 and 19054003) from the Japan Society for the Promotion of Science, NEDO (Japan), and GCOE program GMSI.

\section{REFERENCES}

[1] Li, W. Z., Xie, S. S., Qian, L. X., Chang, B. H., Zou, B. S., Zhou, W. Y., Zhao, R. A., Wang, G., "Large-scale synthesis of aligned carbon nanotubes", Science 1996, 274, 1701.

[2] Rao, C. N. R., Sen, R., Satishkumar, B. C., Govindaraj, A., "Large aligned-nanotube bundles from ferrocene pyrolysis", Chem. Commun. 1998, 1525.

[3] Ren, Z. F., Huang, Z. P., Xu, J. W., Wang, J. H., Bush, P., Siegal, M. P., Provencio, P. N., "Synthesis of large arrays of well-aligned carbon nanotubes on glass", Science 1998, $282,1105$.

[4] Fan, S. S., Chapline, M. G., Franklin, N. R., Tombler, T. W., Cassell, A. M., Dai, H. J., "Self-oriented regular arrays of carbon nanotubes and their field emission properties", Science 1999, 283, 512.

[5] Murakami, Y., Chiashi, S., Miyauchi, Y., Hu, M. H., Ogura, M., Okubo, T., Maruyama, S., "Growth of vertically aligned single-walled carbon nanotube films on quartz substrates and their optical anisotropy", Chem. Phys. Lett. 2004, 385, 298.

[6] Hata, K., Futaba, D. N., Mizuno, K., Namai, T., Yumura, M., Iijima, S., "Water-assisted highly efficient synthesis of impurity-free single-waited carbon nanotubes", Science 2004, 306, 1362.

[7] Zhong, G. F., Iwasaki, T., Honda, K., Furukawa, Y., Ohdomari, I., Kawarada, H., "Very high yield growth of vertically aligned single-walled carbon nanotubes by point-arc microwave plasma CVD", Chemical Vapor Deposition 2005, 11, 127.

[8] Liu, K., Jiang, K. L., Feng, C., Chen, Z., Fan, S. S., "A growth mark method for studying growth mechanism of carbon nanotube arrays", Carbon 2005, 43, 2850.

[9] Li, X., Cao, A. Y., Jung, Y. J., Vjatai, R., Ajayan, P. M., "Bottom-Up Growth of Carbon Nanotube Multilayers: Unprecedented Growth", Nano Letters 2005, 5, 1997.

[10] Mathieu Pinault, Vincent Pichot, Hicham Khodja, Pascale Launois, Ce'cile Reynaud, Mayne-L'Hermite, M., "Evidence of Sequential Lift in Growth of Aligned Multiwalled Carbon Nanotube Multilayers", Nano Lett. 2005.

[11] Zhu, L. B., Xiu, Y. H., Hess, D. W., Wong, C. P., "Aligned carbon nanotube stacks by water-assisted selective etching", Nano Lett. 2005, 5, 2641.

[12] Xiang, R., Luo, G. H., Qian, W. Z., Zhang, Q., Wang, Y., Wei, F., Li, Q., Cao, A. Y., "Encapsulation, compensation, and substitution of catalyst particles during continuous growth of carbon nanotubes", Adv. Mater. 2007, 19, 2360.

[13] Xiang, R., Zhang, Z., Ogura, K., Okawa, J., Einarsson, E., Miyauchi, Y., Shiomi, J., Maruyama, S., "Vertically Aligned 13C Single-Walled Carbon Nanotubes from Noflow Alcohol Chemical Vapor Deposition and their Root Growth Mechanism", Jpn. J. Appl. Phys. 2008, 47, 1971.

[14] Deal, B. E., Grove, A. S., "General relationship for the thermal oxidation of silicon", J. Appl. Phys. 1965, 36, 3770 .

[15] Zhu, L. B., Hess, D. W., Wong, C. P., "Monitoring carbon nanotube growth by formation of nanotube stacks and investigation of the diffusion-controlled kinetics", Journal of Physical Chemistry B 2006, 110, 5445.

[16] Xiang, R., Yang, Z., Zhang, Q., Luo, G. H., Qian, W. Z., Wei, F., Kadowaki, M., Einarsson, E., Maruyama, S., "Growth deceleration of vertically aligned carbon nanotube arrays: Catalyst deactivation or feedstock diffusion controlled?" J. Phys. Chem. C 2008, 112, 4892.

[17] Xiang, R., Einarsson, E., Okawa, J., Thurakitseree, T., Murakami, Y., Shiomi, J., Ohno, Y., Maruyama, S., "Parametric study of ACCVD for controlled synthesis of vertically aligned single-walled carbon nanotubes", $J$. Nanosci. Nanotechnol. in press.

[18] Maruyama, S., Kojima, R., Miyauchi, Y., Chiashi, S., Kohno, M., "Low-temperature synthesis of high-purity single-walled carbon nanotubes from alcohol", Chem. Phys. Lett. 2002, 360, 229.

[19] Xiang, R., Einarsson, E., Okabe, H., Chiashi, S., Shiomi, J., Maruyama, S., "Patterned Growth of High-quality Single-walled Carbon Nanotubes from Dip-coated Catalyst ", Jpn. J. Appl. Phys., submitted.

[20] Xiang, R., Einarsson, E., Okawa, J., Miyauchi, Y., Maruyama, S., "Acetylene-Accelerated Alcohol Catalytic Chemical Vapor Deposition Growth of Vertically Aligned Single-Walled Carbon Nanotubes", J. Phys. Chem. C 2009, 113, 7511 .

[21] Xiang, R., Wu, T. Z., Einarsson, E., Suzuki, Y., Murakami, Y., Shiomi, J., Maruyama, S., "High-Precision Selective Deposition of Catalyst for Facile Localized Growth of Single-Walled Carbon Nanotubes", J. Am. Chem. Soc. 2009, 131, 10344 